\title{
Comparison of the Success Rate of Quadruple and Triple Antibiotic Therapy in Eradicating Helicobacter pylori Infection in Southwest of Iran: A Randomized Clinical Trial
}

\author{
Leila Manzouri, ${ }^{1}$ Farzaneh Zarei, ${ }^{2}$ Narjes Niazi, ${ }^{2}$ and Moslem Sedaghattalab ${ }^{2,}$ \\ ${ }^{1}$ Social Determinants of Health Research Center, Yasuj University of Medical Sciences, Yasuj, Iran \\ ${ }^{2}$ Department of internal medicine, Yasuj University of Medical Sciences, Yasuj, IR Iran \\ "Corresponding author: Moslem Sedaghattalab, Department of Internal Medicine, Imam Sajad Hospital, Yasuj University of Medical Sciences, Yasuj, IR Iran. Tel: \\ +98-9173446077, Fax: +98-7433232173, E-mail: moslem.sedaghattalab@gmail.com
}

Received 2016 December 03; Revised 2016 December 27; Accepted 2016 December 28.

\begin{abstract}
Background: Helicobacter pylori (H. pylori) bacterium has contaminated over $80 \%$ of the population of developing countries. $H$. pylori has a major role in developing gastric ulcer, duodenal ulcer, chronic gastritis, as well as gastric adenocarcinoma and mucosaassociated lymphoid tissue lymphoma (MALT). To eradicate H. pylori infection, information about regional antibiotic resistance is necessary. Therefore, this study was conducted to compare the success rate of quadruple and triple antibiotic therapy in eradicating H. pylori infection in southwest of Iran (Yasuj).

Methods: This was a randomized clinical trial, in which 60 patients aged 15 to 80 years, who referred with dyspepsia and peptic ulcer symptoms to the outpatient clinic of Shahid Mofatteh, Yasuj, Iran, in 2014, enrolled after confirmation of H. pylori infection. All participants provided written informed consent. Then, they were randomly allocated to quadruple (amoxicillin, metronidazole, bismuth subcitrate, and pantoprazole) and triple (amoxicillin, clarithromycin, and pantoprazole) antibiotic therapy regimens for 2 weeks. H. pylori stool antigen test was performed for all patients 6 weeks after the end of the treatment. SPSS17 software was used for data analysis.

Results: From 60 patients, 29 (48.3\%) and 31 (51.7\%) received quadruple and triple antibiotic therapy regimens, respectively. H. pylori stool antigen test was negative in $23(79.3 \%)$ and 16 (51.6\%) treated patients with quadruple and triple antibiotic therapy regimens, respectively $(\mathrm{P}=0.023)$.

Conclusions: The success rate of quadruple therapy was considerably higher than triple antibiotic therapy, and this might be due to clarithromycin resistance.
\end{abstract}

Keywords: Helicobacter pylori, Anti-bacterial Agents, Metronidazole, Clarithromycin, Amoxicillin

\section{Background}

Since the discovery of $H$. pylori by Marshall and Warren in 1982, several studies have been conducted on this topic (1). H. pylori is a bacterium contaminating half of the world's population and is an important cause of gastric cancer (2). H. pylori has contaminated over $80 \%$ of the population in developing countries (2-4). Following gastric colonization, often taking place during childhood, the bacterium has a major role in developing gastric ulcer, duodenal ulcer, chronic gastritis, as well as gastric adenocarcinoma, and mucosa-associated lymphoid tissue lymphoma (MALT) (3). Upper gastrointestinal bleeding is the most prevalent complication of peptic ulcer. H. pylori infection and NSAID (nonsteroidal anti-inflammatory drugs) prescription are 2 independent risk factors of upper gastrointestinal bleeding. Thus, diagnosis and eradication of $H$. pylori is necessary for all patients with upper gastrointestinal bleeding. There is sufficient evidence that $H$. pylori eradication leads to healing of peptic ulcer and decreasing possibility of gastrointestinal re-bleeding (5).

In the recent 2 decades, the standard treatment for $H$. pylori eradication has been the triple regimen including PPI, amoxicillin, clarithromycin, or metronidazole for 7 to 14 days, with the eradication rate of $90 \%$. Although the efficacy of the triple regimen has recently decreased and the eradication rate of $H$. pylori has declined to $<70 \%$ in many countries, increase in microbial resistance, especially resistance to clarithromycin, is the main cause of triple regimen's failure. The quadruple regimen (bismuth based) includes bismuth, tetracycline, metronidazole, and PPI, and it is considered as the first line regimen of choice with $>$ 90\% success rate in case of clarithromycin resistance. Increasing the dose (up to 1500 -1600 mg daily) and duration of the treatment (to 10 - 14 days) with metronidazole can overcome $H$. pylori resistance to metronidazole (3).

After H. pylori treatment, its eradication should be con- 
firmed for all patients, especially for high risk patients including those with peptic ulcer, gastric cancer, and MALTlymphoma. Gastric bleeding due to peptic ulcer would almost never relapse after $H$. pylori eradication. Moreover, $H$. pylori plays a role in developing MALT- lymphoma and its eradication leads to complete recovery in $50 \%$ to $90 \%$ of the cases (6).

Stool antigen test is a precise, non-invasive diagnostic method of detecting $H$. pylori and is conducted through enzyme-linked immunosorbent assay (ELISA) with monoclonal or polyclonal antibody, or through immunochromatographic assay with monoclonal antibodies. The stool antigen test is not accurate in patients with peptic ulcer bleeding and is not recommended for patients with upper gastrointestinal bleeding (5). Monoclonal $H$. pylori stool antigen is an alternative method for detecting the infection in children and adults before and after $H$. pylori eradication (sensitivity: 94\%; specificity: 97\%). The monoclonal stool antigen method is preferred to the polyclonal. Several studies $(n=12)$ have evaluated the efficiency of stool antigen in eradicating $H$.pylori and have demonstrated 93\% sensitivity and $96 \%$ specificity rates (6).

With regards to the importance of $H$.pylori eradication, physicians should be aware of the regional antibiotic resistance pattern prior to prescription of experimental antibiotics. Thus, the present study was conducted to compare the success rate of quadruple and triple antibiotic therapy in eradicating $H$. pylori infection in southwest of Iran (Yasuj).

\section{Methods}

This randomized clinical trial was registered in the Iranian registry of clinical trial, with registration number of ID: IRCT2015050622118N1. This project was approved by the ethics committee of Yasuj University of Medical Sciences (ethical code: 93.06.1.07). Assumed ethical considerations included maintaining the confidentiality of information, allocating a code to each participant, and imposing no costs to patients. In this study, 60 patients, aged 15 to 80 years, with dyspepsia and peptic ulcer symptoms with confirmed H. pylori infection, who reffered to Shahid Mofatteh clinic of Yasuj University of Medical Sciences (Iran) in 2014, were included. Then, a comprehensive explanation about the aim of the study was provided to the patients and written informed consent was obtained. Other inclusion criteria were negative history of $H$. pylori eradication treatment, gastrointestinal bleeding, chronic pulmonary, liver or renal diseases, gastric surgery, pregnancy, recent consumption of NSAIDs (Non Steroid Anti Inflammatory Drugs), taking antibiotics in the last month, taking PPI (proton pump inhibitors), and $\mathrm{H} 2$ receptor antagonists in the previous week. H. pylori infection was confirmed through serology, stool antigen, and endoscopy plus biopsy in 25\%, $15 \%$, and $60 \%$ of patients, respectively. Then, patients were randomly allocated to quadruple (amoxicillin $1000 \mathrm{mg}$ bid, metronidazole $500 \mathrm{mg}$ bid, bismuth subcitrate 2 tablets bid, pantoprazole $40 \mathrm{mg}$ bid) and triple (amoxicillin 1000 $\mathrm{mg}$ bid, clarithromycin $500 \mathrm{mg}$ bid, pantoprazole $40 \mathrm{mg}$ bid) antibiotic therapy regimens for 2 weeks.

$H$. pylori stool antigen test was performed for all patients 6 weeks after the termination of treatment. The test was performed using Liming Bio kit, made in South Korea. The kits were preserved at $2-30^{\circ} \mathrm{C}$ and tests were performed immediately after collecting stool samples.

\subsection{Statistical Analysis}

The sample size was calculated considering Fakheri et al. study that showed $H$. pylori eradication rate of $87 \%$ and $53 \%$ for quadruple and triple treatment, similar to the regimens in our study (7) (power $80 \%$ and $\alpha=0.05$ ). The sample size was 25 per group. Considering the attrition rate of $10 \%$ per group, total sample size was assumed to be 60 patients. The data were analyzed in SPSS software Version 17 (SPSS Inc., Chicago, IL, USA) using Chi-square test and t test. Eradication rate was reported based on intention to treat (ITT) analyses. Statistical significance was set at $\mathrm{P}<0.05$.

\section{Results}

From 60 patients, 29 (48.3\%) and 31 (51.7\%) received quadruple and triple antibiotic therapy regimens, respectively (Figure 1). Of the participants, 23 (38.33\%) and 37 (61.67\%) were male and female, respectively. The mean age of the patients in quadruple and triple antibiotic therapy was $36.03 \pm 12.74$ and $37.09 \pm 11.45$, respectively $(\mathrm{P}=0.73)$ (Table 1).

Table 1. Baseline Characteristics of Patients in Two Groups of Treatment

\begin{tabular}{|c|c|c|c|}
\hline Variable & $\begin{array}{l}\text { Group A ( Triple } \\
\text { Therapy) }\end{array}$ & $\begin{array}{c}\text { Group B } \\
\text { (Quadruple } \\
\text { Therapy) }\end{array}$ & P Value $^{\mathrm{b}}$ \\
\hline $\begin{array}{l}\text { Age ( mean } \pm \\
\text { SD) })^{\mathbf{a}}\end{array}$ & $37.09 \pm 11.45$ & $36.03 \pm 12.74$ & 0.73 \\
\hline \multicolumn{4}{|l|}{ Sex } \\
\hline Male & $12(38.7 \%)$ & $11(37.9 \%)$ & 0.95 \\
\hline Female & $19(61.3 \%)$ & $18(62.1 \%)$ & \\
\hline
\end{tabular}

${ }^{\mathrm{a}} \mathrm{SD}$ : Standard deviation.

${ }^{\mathrm{b}}$ Independent samples T-test and Chi-square test were used for analyses.

Stool antigen test in 23 (79.3\%) and 16 (51.6\%) treated patients with quadruple and triple antibiotic therapy regimens were negative, respectively $(\mathrm{P}=0.023)$ (Table 2 ). 


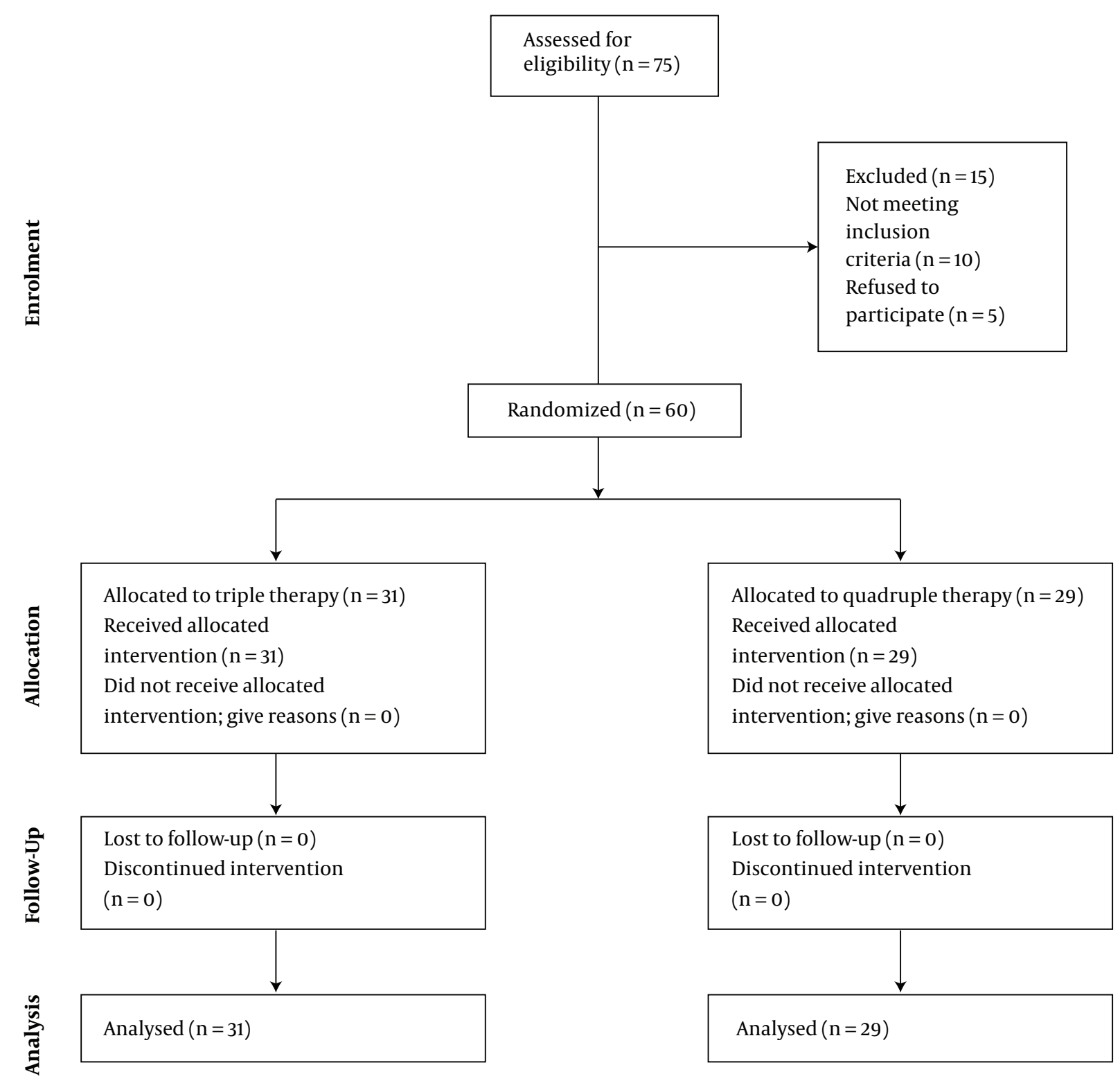

Figure 1. Consort Statement Flow Diagram

\section{Discussion}

The goal of anti- $H$. pylori therapeutic regimens is to obtain a safe, tolerable, economically affordable, and acceptable eradication rate. An anti- H.pylori regimen should lead to at least $80 \%$ to $85 \%$ eradication rate $(8,9)$.

The result of our study revealed that $H$. pylori eradication rate was significantly higher in quadruple antibiotic therapy versus triple antibiotic therapy group. Thus, it was nearly close to an acceptable eradication rate. The result of our study was nearly similar to that of Venerito et al., with higher eradication rate in bismuth-containing quadruple (77.6\%) versus standard triple therapy (67\%) (10). Our finding about eradication rate in quadruple regimen was also consistent with that of Masoodi et al. (79\% and 82.3\% based on the intention to treat and per protocol analyses, respectively) (11). In a review by Fakheri et al. in west Asia, with the same therapeutic regimens as our study, eradication rate varied from $50 \%$ to $87 \%$ and $53 \%$ to $72 \%$ in quadruple and triple therapy, respectively, based on intention to treat analyses (7). In another study of Hosseini et al., eradication 


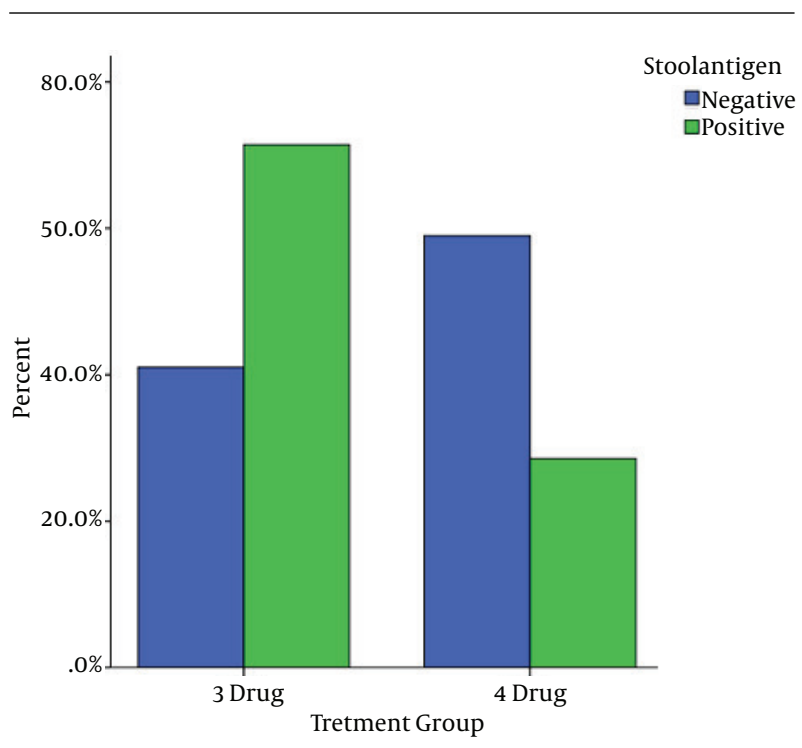

Figure 2. Comparison of Eradication Rate in Triple and Quadruple Therapy Based on Stool Antigen Test

Table 2. Comparison the H. pylori Eradication Rate in Triple and Quadruple Antibiotic Therapy Based on Stool Antigen Test

\begin{tabular}{rccc}
\hline Test & $\begin{array}{c}\text { Group A(Triple } \\
\text { Therapy) }\end{array}$ & $\begin{array}{c}\text { Group B } \\
\text { (Quadruple } \\
\text { Therapy) }\end{array}$ & PValue $^{\mathbf{a}}$ \\
\hline Stool antigen & & & \\
Negative & $16(51.6 \%)$ & $23(79.3 \%)$ & 0.023 \\
Positive & $15(48.4 \%)$ & $6(20.7 \%)$ & \\
\hline
\end{tabular}

${ }^{\mathrm{a}}$ Chi-square test was used for analyses $($ value $=5.053, \mathrm{df}=1$ ).

rate was higher in quadruple therapy (89\%) compared with triple therapy (71\%) (12). Likewise, the results of Ghadir et al. showed higher eradication rate in quadruple therapy (67.4\% vs 51.2\%) (13); although it was lower than our result, it was an acceptable eradication rate.

Antibiotic resistance is a serious obstacle for $\mathrm{H}$. pylori eradication. Geographical location, age, sex, and race are related resistance factors of $H$. pylori bacterium. Thus, detecting antibiotic sensitivity in every region is an important factor in eradicating H. pylori. Resistance to clarithromycin and metronidazole has significantly increased in the recent years $(7,14)$, but amoxicillin resistance is generally low; and in Iran (<10\%), (7) it is lower than Japan, USA, Europe, and China. Wasteful using of clarithromycin in respiratory infections and metronidazole in gynecological and parasitic infections can considerably cause H. pylori resistant to these antibiotics (2).

According to the Malfertheiner P. report, standard triple therapy including a PPI, clarithromycin, and amoxi- cillin is recommended for countries with less than $20 \%$ resistance to clarithromycin; moreover, bismuth-containing quadruple therapy is recommended for regions with high clarithromycin resistance (15).

Resistance to clarithromycin reported from different parts of west Asia ranged from 1.4\% to 26.5\% in Iran (16-20), $16.4 \%$ to $48.2 \%$ in Turkey, $4 \%$ in Lebanon, 33\% in Bahrain, $4 \%$ to $21 \%$ in Saudi Arabia, $12 \%$ in Syria, and $4 \%$ in United Arab Emirates.7 Resistance to clarithromycin has been reported from Japan (9.1\%), USA (6.1\% - 12.6\%), and Europe (15\%) (2) as well. Thus, according to a significantly lower eradication rate of standard triple therapy compared to quadruple therapy and unacceptable eradication rate in our study, it seems that resistance to clarithromycin might be high in our region, located in southwest of Iran, like other provinces such as Tehran, East Azerbaijan, Mashhad, and Hamadan (16-20). Furthermore, clarithromycin is an expensive drug in Iran, and it has been proven that success rate with standard triple therapy would be lower than $90 \%$, when the level of resistance to clarithromycin is more than $10 \%$ (7). Accordingly, triple standard therapy would not be an appropriate regimen for eradication of $H$. pylori infection in our region.

In several studies, resistance to metronidazole has been investigated in different parts of west Asia, and it was found to vary from $33 \%$ to $77 \%$ in Iran $(8,14), 42.6 \%$ in Turkey, 29.5\% in Lebanon, $57 \%$ in Bahrain, 38\% to 80\% in Saudi Arabia, and 63\% in United Arab Emirates (7). H.pylori resistance to metronidazole was $1 \%$ to $12 \%, 20 \%$ to $40 \%, 50 \%$ to $80 \%$, $70 \%$ to $80 \%$, and $80 \%$ in Japan, USA, Europe, Mexico, and Africa, respectively (20) According to a significantly higher eradication rate in quadruple therapy that was near to accepted rate of H.pylori eradication, it seems that resistance to metronidazole in our region is low unlike other parts of Iran (7). Compliance to treatment, smoking (7), and body mass index (1) are also other factors influencing the response to treatment that should be considered. There were some limitations to our study that should be addressed. Being monocenteric, and not checking smoking status and body mass index were limitations of our study.

\subsection{Conclusion}

Quadruple therapy is a better treatment choice compared to triple therapy for eradication of $H$. pylori in southwest of Iran, Yasuj.

\section{Acknowledgments}

We would like to thank Dr. Arash Arya, Dr. Hadi Ghafari, and Dr. Reza Ghaderi for their contributions to this study. 


\section{Footnotes}

Authors' Contribution: Study concept and design: Leila Manzouri, Moslem Sedaghattalab, Farzaneh Zarei; acquisition of data: Narjes Niazi; Analysis and interpretation of data: Leila Manzouri, Farzaneh Zarei, Narjes Niazi, and Moslem Sedaghattalab; and critical revision of the manuscript for important intellectual content: Moslem Sedaghattalab.

Conflict of Interest: There was no conflict of interest.

Funding/Support: This project has been performed under financial support of the Vice Chancellor for Research of Yasuj University of Medical Sciences.

\section{References}

1. Song M, Ang TL. Second and third line treatment options for. World J Gastroenterol. 2014;20(6):1517-28. doi: 10.3748/wjg.v20.i6.1517. [PubMed: 24587627].

2. Picoli SU, Mazzoleni LE, Fernandez H, De Bona LR, Neuhauss E, Longo $\mathrm{L}$, et al. Resistance to amoxicillin, clarithromycin and ciprofloxacin of. Rev Inst Med Trop Sao Paulo. 2014;56(3):197-200. [PubMed: 24878996].

3. Papastergiou V, Georgopoulos SD, Karatapanis S. Treatment of. World J Gastroenterol. 2014;20(29):9898-911. doi: 10.3748/wjg.v20.i29.9898. [PubMed: 25110420].

4. John DV. Peptic ulcer disease and related disorders. In: Longo DL, Kasper DL, Jameson JL, Fauci AS, Hauser SL, Loscalzo J, editors. Harrison's Principles of Internal Medicine. New York: Mc Graw Hill; 2012. p. 2442.

5. Huang TC, Lee CL. Diagnosis, treatment, and outcome in patients with bleeding peptic ulcers and. Biomed Res Int. 2014;2014:658108. doi: 10.1155/2014/658108. [PubMed: 25101293].

6. Rollan A, Arab JP, Camargo MC, Candia R, Harris P, Ferreccio C, et al. Management of. World J Gastroenterol. 2014;20(31):10969-83. doi: 10.3748/wjg.v20.i31.10969. [PubMed: 25152601].

7. Fakheri H, Bari Z, Aarabi M, Malekzadeh R. eradication in West Asia: a review. World J Gastroenterol. 2014;20(30):10355-67. doi: 10.3748/wjg.v20.i30.10355. [PubMed: 25132752].
8. Keshavarz AA. Omeprazole-based triple therapy with low-versus high-dose of clarithromycin plus amoxicillin forH pylorieradication in Iranian population. World J Gastroentero. 2007;13(6):930. doi: 10.3748/wjg.v13.i6.930.

9. Vafaeimanesh J, Jalalzadeh M, Nazarian M. Expression comparison of azithromycin and clarithromycin in triple therapy regimens for eradication of. Saudi J Kidney Dis Transpl. 2014;25(1):53-7.

10. Venerito M, Krieger T, Ecker T, Leandro G, Malfertheiner P. Metaanalysis of bismuth quadruple therapy versus clarithromycin triple therapy for empiric primary treatment of. Digestion. 2013;88(1):33-45. doi: 10.1159/000350719. [PubMed: 23880479].

11. Masoodi M, Panahian M, Rezadoost A, Heidari A. Eradication Rate of. Middle East J Dig Dis. 2013;5(2):81-5. [PubMed: 24829674].

12. Hosseini SM, Sharifipoor F, Nazemian F, Ghanei H, Zivarifar HR, Fakharian T. eradication in renal recipient: triple or quadruple therapy? Acta Med Iran. 2014;52(4):271-4. [PubMed: 24901856].

13. Ghadir MR, Shafaghi A, Iranikhah A, Pakdin A, Joukar F, MansourGhanaei F. Furazolidone, amoxicillin and omeprazole with or without bismuth for eradication of. Turk J Gastroenterol. 2011;22(1):1-5. [PubMed: 21480103].

14. Khoshnood A, Hakimi P, Salman-Roghani H, Reza Mirjalili M. Replacement of clarithromycin with azithromycin in triple therapy regimens for the eradication of. J Med Life. 2014;7(2):254-9. [PubMed: 25408735].

15. Malfertheiner P, Megraud F, O'Morain CA, Atherton J, Axon AT, Bazzoli F, et al. Management of. Gut. 2012;61(5):646-64. doi: 10.1136/gutjnl2012-302084. [PubMed: 22491499].

16. Majlesi A, Khorasani MS, Aslani MM, Alikhani MY. Antibiotic susceptibility of. Inter J Enteric Pathogens. 2013;1(1):8-11.

17. Milani M, Ghotaslou R, Akhi MT, Nahaei MR, Hasani A, Somi MH, et al. The status of antimicrobial resistance of. J Infect Chemother. 2012;18(6):848-52. doi:10.1007/s10156-012-0425-4. [PubMed: 22581031].

18. Mohammadi M, Doroud D, Mohajerani N, Massarrat S. antibiotic resistance in Iran. World J Gastroenterol. 2005;11(38):6009-13. [PubMed: 16273615].

19. Shokrzadeh L, Jafari F, Dabiri H, Baghaei K, Zojaji H, Alizadeh $\mathrm{AH}$, et al. Antibiotic susceptibility profile of. Saudi J Gastroenterol. 2011;17(4):261-4. doi: 10.4103/1319-3767.82581. [PubMed: 21727733].

20. Zendedel A, Moradimoghadam F, Almasi V, Zivarifar H. Antibiotic resistance of. J Pak Med Assoc. 2013;63(3):336-9. [PubMed: 23914633]. 